\title{
THE INDUCED SUBGRAPH OF THE UNITARY CAYLEY GRAPH OF A COMMUTATIVE RING OVER REGULAR ELEMENTS
}

\author{
ALI REZA NAGHIPOUR \\ Received 22 December, 2014
}

\begin{abstract}
Let $R$ be a finite commutative ring and $R^{*}$ be the group of units of $R$. The unitary Cayley graph of $R$, denoted by $G(R)$, is the graph obtained by setting all the elements of $R$ to be the vertices and defining vertices $x$ and $y$ to be adjacent if and only if $x-y \in R^{*}$. We denote by $\Gamma(R)$ the induced subgraph of $G(R)$ whose vertex set is $R^{*}$. In this paper, the basic properties of $\Gamma(R)$ are investigated and some characterization results regarding connectedness, chromatic number, chromatic index, girth and planarity of $\Gamma(R)$ are given. In addition, by using the concept of spectrum of graphs, it is shown that for two finite commutative rings $R$ and $S$, if $\Gamma(R)$ is non empty, then $\Gamma(R) \cong \Gamma(S)$ as graphs if and only if $R / J(R) \cong S / J(R)$ as rings, where $J(R)$ and $J(S)$ are the Jacobson radicals of $R$ and $S$, respectively. As a consequence of this result, we obtain that the structure of a reduced ring $R$ can be determined by $\Gamma(R)$.
\end{abstract}

2010 Mathematics Subject Classification: 05C25; 05C40; 13M05; $16 \mathrm{U} 60$

Keywords: Connectivity, diameter, girth, planar graphs, chromatic number, chromatic index, isomorphisms

\section{INTRODUCTION}

The study of algebraic structures, using the properties of graph associated to them, becomes an interesting topic in the last years. There are many papers on assigning a graph to a ring, for example see [6], [8], [12] and [28]. For additional historical background the reader is referred to the article [24], which also contains references to recent work in this area. Our main object is to establish connections between ring theoretic properties with the properties of the associated graph.

For the sake of completeness, first we state some definitions and notions used throughout to keep this paper as self contained as possible. The graph in this paper are simple, that is they have no loops or multiple edges. For a graph $G$, let $V(G)$ denotes the set of vertices, and let $E(G)$ denotes the set of edges. For $x \in V(G)$ we denote by $N(x)$ the set of all vertices of $G$ adjacent to $x$. Also, the degree of $x$, denoted $d_{G}(x)$, is the size of $N(x)$. The maximum degree of $G$ denoted by $\Delta(G)$. The union of two simple graphs $G$ and $H$ is the graph $G \cup H$ with the vertex set $V(G) \cup V(H)$ and the edge set $E(G) \cup E(G)$. If $G$ and $H$ are disjoint, we refer 
to their union as a disjoint union, and denote it by $G+H$. The disjoint union of $n$ copies of $G$ is denoted by $n G$.

Let $G$ be a graph. For two vertices $x$ and $y$ of $G$, a path of length $n$ between $x$ and $y$ is an ordered list of distinct vertices $x=x_{0}, x_{1}, \ldots, x_{n}=y$ such that $x_{i-1}$ is adjacent to $x_{i}$ for $i=1, \ldots, n$. The distance between $x$ and $y$, denoted by $d(x, y)$, is the length of shortest path between $x$ and $y(d(x, x)=0$ and $d(x, y)=\infty$ if there is no path between $x$ and $y$ ). The largest distance among all distances between pairs of the vertices of a graph $G$ is called the diameter of $G$ and is denoted by $\operatorname{diam}(G)$. A cycle in $G$ is a path that begins and ends at the same vertex. The girth of $G$, denoted by $\operatorname{gr}(G)$, is the length of a shortest cycle in $G(\operatorname{gr}(G)=\infty$ if $G$ has no cycles). A graph $G$ is called connected if for any vertices $x$ and $y$ of $G$ there is a path between $x$ and $y$. Otherwise, $G$ is called disconnected. A graph with no edges is called an empty graph.

A graph in which each pair of distinct vertices is joined by an edge is called complete graph. We denote the complete graph on $n$ vertices by $K_{n}$. Let $r$ be a positive integer. A graph is called $r$-regular, if all its vertices have degree $r$. A graph is called $r$-partite if the vertex set admits a partition into $r$ classes such that vertices in the same partition class are not adjacent. A 2-partite graph is also called bipartite graph. An $r$-partite graph is called complete if every two vertices in different parts are adjacent. A complete $r$-partite graph with part sizes $m_{1}, m_{2}, \ldots, m_{r}$ is denoted by $K_{m_{1}, m_{2}, \ldots, m_{r}}$.

Throughout this paper, all rings are assumed to be finite commutative with nonzero identity. For a ring $R$, we denote by $R^{*}$ and $J(R)$ the group of units of $R$ and the Jacobson radical of $R$, respectively. For a set $X,|X|$ denotes the cardinal of $X$. Also, $\mathbb{F}_{p^{n}}$ denotes the field with $p^{n}$ elements and $\mathbb{Z}_{n}$ denotes for the ring of integers modulo $n$ and following the literature, we write

$$
D_{2}(R)=\left\{\left(\begin{array}{cc}
a & b \\
0 & a
\end{array}\right) \mid a, b \in R\right\} .
$$

It is well known that every finite commutative ring can be expressed as a direct product of finite local rings, and this decomposition is unique up to permutations of such local rings (see [7, Theorem 8.7]). Throughout the paper we assume the following:

Assumption 1. $R=R_{1} \times \cdots \times R_{n}$ is a finite commutative ring, where $R_{i}$ is a local ring with maximal ideal $\mathfrak{m}_{i}$ of order $m_{i}, 1 \leq i \leq n$. We assume $\pi_{i}: R_{i} \longrightarrow R_{i} / \mathfrak{m}$ is the canonical map and $f_{i}=\left|R_{i} / \mathfrak{m}_{i}\right|$ and

$$
f_{1} \leq f_{2} \leq \ldots \leq f_{n} .
$$

For other notations not mentioned in the paper, one can refer to [7] and [30].

The Cayley graph introduced by Arthur Cayley in 1878 is a useful tool for connection between group theory and the theory of algebraic graphs. The unitary Cayley 
graph of a ring $R$, denoted by $G(R)$, is the graph whose vertex set is $R$, and in which two vertices $x$ and $y$ are adjacent if and only if $x-y \in R^{*}$. For some other recent papers on unitary Cayley graphs, see [1], [2] and [4]. Let $\Gamma(R)$ be the induced subgraph of $G(R)$ whose vertex set is $R^{*}$. This paper studies the graph $\Gamma(R)$ and the main motivation is the fact that a reduced ring $R$ can be determined by $\Gamma(R)$ (see Corollary 6.4). The graphs $\Gamma\left(\mathbb{Z}_{2}\right), \Gamma\left(\mathbb{Z}_{3}\right), \Gamma\left(\mathbb{Z}_{5}\right)$ and $\Gamma\left(\mathbb{Z}_{3} \times \mathbb{Z}_{3}\right)$ are presented in Figure 1.

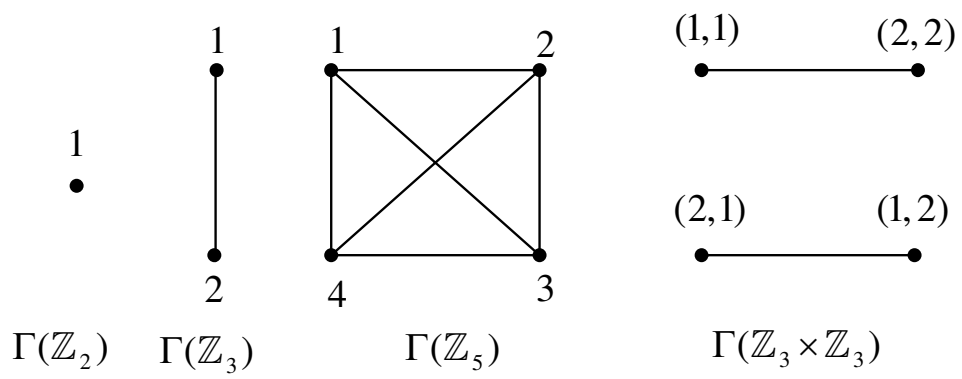

FIGURE 1.

The plan of the paper is as follows: In Section 2, we give some basic properties of $\Gamma(R)$. In Section 3, we determine the number of component of $\Gamma(R)$. Also, we completely characterize the diameter and girth of $\Gamma(R)$. In Section 4, we give a necessary and sufficient condition for the planarity of $\Gamma(R)$. In Section 5, some characterization results regarding chromatic number and chromatic index of $\Gamma(R)$ are given. For two finite rings $R$ and $S$, the question of when $\Gamma(R) \cong \Gamma(S)$ as graphs, implies that $R \cong S$ as rings is very interesting and this kind of question has been studied extensively in [3], [5] and [18]. In the final section, by using the concept of spectrum of graphs, we show that, if $\Gamma(R)$ is non empty, then $\Gamma(R) \cong \Gamma(S)$ as graphs, if and only if $R / J(R) \cong S / J(R)$ as rings.

\section{SOME BASIC PROPERTIES OF $\Gamma(R)$}

In this section we study some basic properties of $\Gamma(R)$. The following proposition is well-known, but a simple proof of it is included here for the sake of completeness.

Proposition 1. Let $R$ be a finite local ring with maximal ideal $\mathfrak{m}$. Then there exists a prime number $p$ such that $|R / \mathfrak{m}|,|R|$ and are all power of $p$.

Proof. For a group $G$, we denote the order of an element $x \in G$ by $\operatorname{ord}_{G}(x)$. Since $F=R / \mathfrak{m}$ is a field, there exists a prime number $p$ such that $|F|$ is a power of $p$. Let $n=|R|$ and $q$ be a prime divisor of $n$. Then there exists $x \in R$ such that $\operatorname{ord}_{R}(x)=q$. Since $\operatorname{ord}_{F}(x+\mathfrak{m})$ is a power of $p$ and divides $q$, we must have $q=p$. Therefore $|R|$ is a power of $p$, which implies that $|\mathfrak{m}|$ is also a power of $p$ 
The direct product (sometimes called tensor product or category product) of two graphs $G$ and $H$, denoted by $G \times H$, is the graph with vertex set $V(G) \times V(H)$ specified by putting $\left(x_{1}, y_{1}\right)$ adjacent to $\left(x_{2}, y_{2}\right)$ if and only if $x_{1}$ is adjacent to $x_{2}$ in $G$ and $y_{1}$ is adjacent to $y_{2}$ in $H$.

Theorem 1. Let $R$ be as in Assumption 1. Then

(1) $\Gamma(R)$ is a $\prod_{i=1}^{n}\left(\left|R_{i}\right|-2\left|\mathfrak{m}_{i}\right|\right)$-regular graph,

(2) $\Gamma(R) \cong \Gamma\left(R_{1}\right) \times \cdots \times \Gamma\left(R_{n}\right)$.

Proof. (1) Let $1 \leq i \leq n$ and $R_{i} / \mathfrak{m}_{i}=\left\{\mathfrak{m}_{i}, a_{i 1}+\mathfrak{m}_{i}, \ldots, a_{i\left(f_{i}-1\right)}+\mathfrak{m}_{i}\right\}$. If $x_{i}$ is an arbitrary element of $R_{i}^{*}$, then there exists $1 \leq k \leq f_{i}-1$ such that $x_{i} \in a_{i k}+\mathfrak{m}_{i}$. Therefore $N\left(x_{i}\right)=\cup_{j \neq k}\left(a_{i j}+\mathfrak{m}_{i}\right)$ and so $d_{\Gamma\left(R_{i}\right)}\left(x_{i}\right)=\left(f_{i}-2\right)\left|\mathfrak{m}_{i}\right|=\left|R_{i}\right|-2\left|\mathfrak{m}_{i}\right|$. It follows that $d_{\Gamma(R)}\left(x_{1}, \ldots, x_{n}\right)=\prod_{i=1}^{n}\left(\left|R_{i}\right|-2\left|\mathfrak{m}_{i}\right|\right)$.

(2) The assertion follows easily from the fact that $R^{*}=R_{1}^{*} \times \cdots \times R_{n}^{*}$.

Theorem 2. Let $(R, \mathfrak{m})$ be a local ring. Then

(1) $\Gamma(R)$ is a complete multipartite graph whose partite sets are the set of all cosets of $\mathfrak{m}$ except $\mathfrak{m}$,

(2) $\Gamma(R)$ is a complete bipartite graph if and only if $|R / \mathfrak{m}|=3$.

Proof. (1) Let $|R / \mathfrak{m}|=f$ and $R / \mathfrak{m}=\left\{\mathfrak{m}, a_{1}+\mathfrak{m}, \ldots, a_{f-1}+\mathfrak{m}\right\}$. Then $\Gamma(R)$ is complete bipartite with partites $a_{1}+\mathfrak{m}, \ldots, a_{f-1}+\mathfrak{m}$.

(2) Let $|R / \mathfrak{m}|=3$. Then it is easy to see that $\Gamma(R)$ is complete bipartite.

If $|R / \mathfrak{m}|=2$, then $\Gamma(R)$ is non empty and hence $\Gamma(R)$ is not complete bipartite graph. If $|R / \mathfrak{m}|=n$, where $n>3$, then $\Gamma(R)$ is a complete $n-1$ partite graph and hence $\Gamma(R)$ graph is not complete bipartite graph. Therefore if $\Gamma(R)$ is a complete bipartite graph, then $|R / \mathfrak{m}|=3$ and the proof is completed.

Theorem 3. Let $R$ be a finite ring. Then

(1) $\Gamma(R)$ has only one vertex if and only if $R \cong \mathbb{Z}_{2}^{n}$ for some natural number $n$,

(2) $\Gamma(R)$ has only two vertices if and only if $R \cong \mathbb{Z}_{2}^{n} \times S$, where $n$ is a non negative integer, $S \cong \mathbb{Z}_{3}, S \cong \mathbb{Z}_{4}$ or $S \cong D_{2}\left(\mathbb{Z}_{2}\right)$

Proof. (1) Let $R$ be as in Assumption 1. If $\Gamma(R)$ has only one vertex, then $\left|R^{*}\right|=$ $\left|R_{1}^{*}\right| \times \cdots \times\left|R_{n}^{*}\right|=1$. Therefore $\left|R_{i}^{*}\right|=\left|R_{i}\right|-\left|\mathfrak{m}_{i}\right|=1$ for each $i$. It follows from Proposition 1 that $\left|\mathfrak{m}_{i}\right|=1$ and hence $\left|R_{i}\right|=2$. Therefore $R \cong \mathbb{Z}_{2}^{n}$ for some natural number $n$. The converse is easily.

(2) Follows easily from [11, Corollary 4.5].

\section{CONNECTIVITY, DIAMETER AND GIRTH}

We begin with the following theorem. 
Theorem 4. Let $R$ be as in Assumption 1. Then the following are equivalent:

(1) $\Gamma(R)$ is non empty,

(2) $f_{1} \neq 2$,

(3) $R / J(R)$ does not contain a summand isomorphic to $\mathbb{Z}_{2}$,

(4) every element of $R$ can be expressed as the sum of two units.

Proof. (1) $\Longrightarrow$ (2) Trivial.

(2) $\Longrightarrow$ (1) Let $f_{i} \neq 2$ for all $1 \leq i \leq n$. Then $f_{1} \geq 3$ and hence there exists two elements $a=\left(a_{1}, \ldots, a_{n}\right)$ and $b=\left(b_{1}, \ldots, b_{n}\right)$ of $R^{*}$ such that $a_{i}-b_{i} \notin \mathfrak{m}_{i}$ for all $1 \leq i \leq n$. Therefore $a$ and $b$ are two adjacent elements of $\Gamma(R)$, and hence $\Gamma(R)$ is non empty.

(3) $\Longleftrightarrow(2)$ We have $R / J(R) \cong R_{1} / \mathfrak{m}_{1} \times \cdots \times R_{n} / \mathfrak{m}_{n}$. By uniqueness of WedderburnArtin Theorem ([21, Theorem 3.5]), we have that $f_{1}=2$ if and only if $R / J(R)$ has a summand isomorphic to $\mathbb{Z}_{2}$.

(3) $\Longleftrightarrow$ (4) Follows from [22, Theorem 1].

Theorem 5 ([17, Theorem 5.29]). Let $G$ and $H$ be graphs with at least one edge. Then $G \times H$ is connected if and only if both $G$ and $H$ are connected and at least one of them is non bipartite. Furthermore, if both $G$ and $H$ are connected and bipartite, then $G \times H$ has exactly two components.

Theorem 6. Let $R$ be as in Assumption 1. Then $\Gamma(R)$ is disconnected if and only if $f_{1}=2$ or $f_{n}=3$.

Proof. Follows easily from Theorems 2(2), 4 and 5.

The following theorem determine the number of components of $\Gamma(R)$. For a graph $G$ we write $k(G)$ for the number of components of $G$.

Theorem 7. Let $R$ be as in Assumption 1. Then

$$
k(\Gamma(R))= \begin{cases}\left|R^{*}\right| & \text { if } f_{1}=2, \\ 2^{r-1} & \text { if } f_{1}=\cdots=f_{r}=3 \text { and } f_{r+1}>3, \\ 2^{n-1} & \text { if } f_{1}=\cdots=f_{n}=3, \\ 1 & \text { otherwise. }\end{cases}
$$

Proof. If $f_{1}=2$, then $\Gamma(R)$ is an empty graph and hence $k(\Gamma(R))=\left|R^{*}\right|$.

If $f_{1}=\cdots=f_{n}=3$, then we see easily by induction that

$$
\Gamma(R) \cong K_{m_{1}, m_{1}} \times \cdots \times K_{m_{n}, m_{n}} \cong 2^{n-1} K_{m_{1} \ldots m_{n}, m_{1} \ldots m_{n}} .
$$

So $k(\Gamma(R))=2^{n-1}$.

Now let there exists $1 \leq r<n$ such that $f_{1}=\cdots=f_{r}=3$ and $f_{r+1}>3$. For convenience, we will take $S=R_{r+1} \times \cdots \times R_{n}$. Then

$$
\Gamma(R) \cong K_{m_{1}, m_{1}} \times \ldots \times K_{m_{r}, m_{r}} \times \Gamma(S) \cong 2^{r-1} K_{m_{1} \ldots m_{r}, m_{1} \ldots m_{r}} \times \Gamma(S) .
$$

Since $\Gamma(S)$ is a connected non bipartite graph, Theorem 5 implies that $k(\Gamma(R))=$ $2^{r-1}$. 
If $f_{1}>3$, then by Theorem $6, \Gamma(R)$ is connected and hence $k(\Gamma(R))=1$. This completes the proof.

The following theorem shows that the diameter of $\Gamma(R)$ has five possibilities.

Theorem 8. Let $R$ be as in Assumption 1 and $|R|>2$. Then

$$
\operatorname{diam}(\Gamma(R))= \begin{cases}1 & \text { if } R \text { is a field, } \\ 0 & \text { if } f_{n}=2, \\ \infty & \text { if } f_{n}>f_{1}=2 \text { or } f_{1}=f_{2}=3, \\ 3 & \text { if } f_{1}=3, f_{2} \geq 4 \\ 2 & \text { otherwise. }\end{cases}
$$

Proof. Let $a=\left(a_{1}, a_{2}, \ldots, a_{n}\right)$ and $b=\left(b_{1}, b_{2}, \ldots, b_{n}\right)$ be two arbitrary distinct vertices of $\Gamma(R)$. We consider the following cases:

(1) $R$ is a field. Since $|R|>2, \Gamma(R)$ is isomorphic to $K_{|R|-1}$ and hence $\operatorname{diam}(\Gamma(R))=1$.

(2) $f_{n}=2$. Then by Theorem $3(1), \Gamma(R)$ has only one vertex and hence $\operatorname{diam}(\Gamma(R))=0$.

(3) $f_{n}>f_{1}=2$ or $f_{1}=f_{2}=3$. Then by Theorem 2, $\Gamma(R)$ is disconnected and hence $\operatorname{diam}(\Gamma(R))=\infty$.

(4) Now let $n \geq 2, f_{1}=3$ and $f_{2} \geq 4$. We consider two cases:

Case 1: $\pi_{1}\left(a_{1}\right)=\pi_{1}\left(b_{1}\right)$. In this case, For each $1 \leq i \leq n$, we choose a vertex $c_{i}$ of $\Gamma\left(R_{i}\right)$ such that $\pi_{i}\left(c_{i}\right) \notin\left\{\pi_{i}\left(a_{i}\right), \pi_{i}\left(b_{i}\right)\right\}$. Set $c=\left(c_{1}, c_{2}, \ldots, c_{n}\right)$. Then $a, b \in N(c)$. It follows that $d(a, b)=2$.

Case 2: $\pi_{1}\left(a_{1}\right) \neq \pi_{1}\left(b_{1}\right)$. We consider two subcases:

Subcase 1: If $\pi_{i}\left(a_{i}\right) \neq \pi_{i}\left(b_{i}\right)$ for all $2 \leq i \leq n$, then $a$ and $b$ are adjacent. Therefore $d(a, b)=1$.

Subcase 2: Suppose that $\pi_{i}\left(a_{i}\right)=\pi_{i}\left(b_{i}\right)$ for some $2 \leq i \leq n$. For each $1 \leq$ $i \leq n$, if $\pi_{i}\left(a_{i}\right) \neq \pi_{i}\left(b_{i}\right)$, we set $c_{i}=b_{i}$ and $d_{i}=a_{i}$ and if $\pi_{i}\left(a_{i}\right)=\pi_{i}\left(b_{i}\right)$, we choose $c_{i}, d_{i} \in R_{i}^{*}$ such that $\left|\left\{\pi_{i}\left(a_{i}\right)=\pi_{i}\left(a_{i}\right), \pi_{i}\left(c_{i}\right), \pi_{i}\left(d_{i}\right)\right\}\right|=3$. Now set $c=\left(c_{1}, c_{2}, \ldots, c_{n}\right)$ and $d=\left(d_{1}, d_{2}, \ldots, d_{n}\right)$. Then $a, c, d, b$ is path between $a$ and $b$. Therefore $\operatorname{diam}(\Gamma(R)) \leq 3$. On the other hand, the vertices $a$ and $b$ neither adjacent nor do they have a common neighbor, hence $\operatorname{diam}(\Gamma(R)) \geq 3$. So $\operatorname{diam}(\Gamma(R))=3$.

(5) $n=1, f_{1} \geq 3$ and $R$ is not a field. Then $\Gamma(R)$ is isomorphic to $K_{m_{1}, \ldots, m_{f_{1}-1}}$, where $m_{1}=\ldots=m_{f_{1}-1}=\left|\mathfrak{m}_{1}\right| \geq 2$. It follows that $\operatorname{diam}(\Gamma(R))=2$.

(6) $n \geq 2, f_{1} \geq 4$. Since $f_{i} \geq 4$ for each $1 \leq i \leq n$, we can choose $c_{i} \in R_{i}^{*}$ such that $\pi_{i}\left(c_{i}\right) \notin\left\{\pi_{i}\left(a_{i}\right), \pi_{i}\left(b_{i}\right)\right\}$. Set $c=\left(c_{1}, c_{2}, c_{3}, \ldots, c_{n}\right)$. Then $a, b \in N(c)$. It follows that $d(a, b) \leq 2$. On the other hand, if $x=\left(x_{1}, a_{2}, \ldots, a_{n}\right)$ is a vertex of $\Gamma(R)$ such that $\pi_{1}\left(x_{1}\right) \neq \pi_{1}\left(a_{1}\right)$, then $a$ and $x$ are not adjacent, and so $d(x, a) \geq 2$. Therefore $\operatorname{diam}(\Gamma(R))=2$. This completes the proof.

The following theorem shows that the girth of $\Gamma(R)$ has four possibilities. 
Theorem 9. Let $R$ be as in Assumption 1. Then

$$
\operatorname{gr}(\Gamma(R))=\left\{\begin{array}{l}
\infty \quad \text { if } f_{1}=2, \text { or } R \cong \mathbb{Z}_{3}^{n}, \\
6 \quad \text { if } f_{1}=\ldots=f_{n-1}=3, f_{n}=4, \\
4 \quad \text { if } f_{1}=\ldots=f_{n-1}=3, f_{n} \geq 5, \\
3 \quad \text { otherwise. }
\end{array}\right.
$$

Proof. Consider the following cases:

(1) $f_{1}=2$. Then Theorem 4 implies that $\Gamma(R)$ is non empty and hence $\operatorname{gr}(\Gamma(R))=$ $\infty$.

(2) $f_{1}=\ldots=f_{n-1}=3$ and $f_{n}=4$. In this case, we have

$$
\begin{aligned}
\operatorname{gr}(\Gamma(R)) & =\operatorname{gr}\left(K_{m_{1}, m_{1}} \times \cdots \times K_{m_{n-1}, m_{n-1}} \times K_{m_{n}, m_{n}, m_{n}}\right) \\
& =\operatorname{gr}\left(2^{n-2} K_{m_{1} \ldots m_{n-1}, m_{1} \ldots m_{n-1}} \times K_{m_{n}, m_{n}, m_{n}}\right) \\
& =\operatorname{gr}\left(K_{m_{1} \ldots m_{n-1}, m_{1} \ldots m_{n-1}} \times K_{m_{n}, m_{n}, m_{n}}\right) \\
& =6 .
\end{aligned}
$$

(3) $f_{1}=\ldots=f_{n-1}=3$ and $f_{n} \geq 5$. In this case, since $\Gamma(R)$ is bipartite, we have $\operatorname{gr}(\Gamma(R)) \geq 4$. For each $1 \leq i \leq n-1$, choose elements $a_{i}, b_{i}$ of $R_{i}$ such that $\left|\left\{\pi_{i}\left(a_{i}\right), \pi_{i}\left(b_{i}\right)\right\}\right|=2$. Let $x_{n}, y_{n}, z_{n}, w_{n}$ of $R_{n}$ such that $\left|\left\{\pi_{i}\left(x_{n}\right), \pi_{i}\left(y_{n}\right), \pi_{i}\left(z_{n}\right), \pi_{i}\left(w_{n}\right)\right\}\right|=4$. Now let

$$
\begin{aligned}
x & =\left(a_{1}, a_{2}, \ldots, a_{n-1}, x_{n}\right), \\
y & =\left(b_{1}, b_{2}, \ldots, b_{n-1}, y_{n}\right), \\
z & =\left(a_{1}, a_{2}, \ldots, a_{n-1}, z_{n}\right), \\
w & =\left(b_{1}, b_{2}, \ldots, b_{n-1}, w_{n}\right) .
\end{aligned}
$$

Then $x, y, z, w, x$ is a cycle of length 4 , and hence $\operatorname{gr}(\Gamma(R))=4$.

(4) $f_{1}=\cdots=f_{n}=3$. Then

$$
\Gamma(R) \cong K_{m_{1}, m_{1}} \times \cdots \times K_{m_{n}, m_{n}} \cong 2^{n-1} K_{m_{1} \ldots m_{n}, m_{1} \ldots m_{n}} .
$$

So $\operatorname{gr}(\Gamma(R))=4$ if $m_{1} \ldots m_{n}>1$ and $\operatorname{gr}(\Gamma(R))=\infty$ if $m_{1}=\ldots=m_{n}=1$ (i.e., $\operatorname{gr}(\Gamma(R))=\infty$ if $\left.R \cong \mathbb{Z}_{3}^{n}\right)$.

(5) Finally, suppose that $f_{1} \geq 4$. Let $a_{i}, b_{i}, c_{i}$ be elements of $R_{i}$ such that $\left|\left\{\pi_{i}\left(a_{i}\right), \pi_{i}\left(b_{i}\right), \pi_{i}\left(c_{i}\right)\right\}\right|=3$. Let

$$
\begin{aligned}
a & =\left(a_{1}, a_{2}, \ldots, a_{n}\right), \\
b & =\left(b_{1}, b_{2}, \ldots, b_{n}\right), \\
c & =\left(c_{1}, c_{2}, \ldots, c_{n}\right) .
\end{aligned}
$$

Then $a, b, c, a$ is a cycle of length 3 , and hence $\operatorname{gr}(\Gamma(R))=3$. This completes the proof. 


\section{Planarity}

A graph is said to be planar if it can be drawn in the plane such that its edges intersect only at their ends. A subdivision of an edge is obtained by inserting into this edge some new vertices of degree two. A remarkably simple characterization of planar graphs was given by Kuratowski in 1930. Kuratowski's Theorem says that a graph is planar if and only if it contains no subdivision of $K_{5}$ or $K_{3,3}$ (see [9, Theorem 10.30]). Also if $G$ is a planar graph, then $G$ contains a vertex of degree at most five (see [9, Corollary 10.22]).

The following theorem gives necessary and sufficient condition for the planarity of $\Gamma(R)$.

Theorem 10. Let $R$ be a finite ring. Then $\Gamma(R)$ is planar if and only if $\Gamma(R)$ is empty or $R$ is isomorphic to one of the following rings:

$$
\mathbb{Z}_{3}^{r} \times \mathbb{F}_{4}, \mathbb{Z}_{3}^{r}, \mathbb{Z}_{3}^{r} \times \mathbb{F}_{4} \times \mathbb{F}_{4}, \mathbb{Z}_{3}^{r} \times \mathbb{Z}_{5}, \mathbb{Z}_{3}^{r} \times \mathbb{Z}_{9}, \mathbb{Z}_{3}^{r} \times D_{2}\left(\mathbb{Z}_{3}\right)(r \geq 0) .
$$

Proof. Let $R$ be as in Assumption 1 and let $1 \leq i \leq n$. By Theorem 1, we may assume $\left|R_{i}\right|=p_{i}^{\alpha_{i}}$ and $\left|\mathfrak{m}_{i}\right|=p_{i}^{\beta_{i}}$, where $p$ is a prime number and $\alpha_{i}$ is a natural number and $\beta_{i}$ is a non negative integer. Now consider the following cases:

(1) $p_{i}^{\alpha_{i}}-2 p_{i}^{\beta_{i}}=0$. In this case, we have $f_{i}=2$ and hence $\Gamma\left(R_{i}\right)$ is an empty graph.

(2) $p_{i}^{\alpha_{i}}-2 p_{i}^{\beta_{i}}=1$. In this case, we have $p_{i}=3, \alpha_{i}=1$ and $\beta_{i}=0$. So $\left|R_{i}\right|=3$ and hence $R_{i} \cong \mathbb{Z}_{3}$.

(3) $p_{i}^{\alpha_{i}}-2 p_{i}^{\beta_{i}}=2$ for some $i$. In this case we have $p_{i}=2, \alpha_{i}=2$ and $\beta_{i}=0$. So $\left|R_{i}\right|=4$ and hence $R_{i} \cong \mathbb{F}_{4}$.

(4) $p_{i}^{\alpha_{i}}-2 p_{i}^{\beta_{i}}=3$. If $\beta_{i}=0$, we have $\left|R_{i}\right|=5$ and hence $R_{i} \cong \mathbb{Z}_{5}$. If $\beta_{i} \neq 0$, we have $p_{i}=3, \alpha_{i}=2$ and $\beta_{i}=1$. So $R_{i} \cong \mathbb{Z}_{9}$ or $R_{i} \cong D_{2}\left(\mathbb{Z}_{3}\right)$, by [26, Theorem $3(4)]$.

(5) $p_{i}^{\alpha_{i}}-2 p_{i}^{\beta_{i}}=4$. In this case we have $p_{i}=2$. If $\beta_{i}=0$, then $2^{\alpha_{i}-1}=3$, which is a contradiction. So we must have $\beta_{i} \neq 0$. Therefore $\alpha_{i}=3$ and $\beta_{i}=1$, which is again a contradiction by [26, Theorem 3(1)].

(6) $p_{i}^{\alpha_{i}}-2 p_{i}^{\beta_{i}}=5$. If $\beta_{i} \neq 0$, then $p_{i}=5$ and hence $5^{\alpha_{i}-1}=3$, which is a contradiction. So, in this case we have $\beta_{i}=0$. Therefore $\alpha_{i}=1$ and so $R_{i} \cong \mathbb{Z}_{7}$. (Note that $\Gamma\left(\mathbb{Z}_{7}\right)$ is not planar).

Since $\Gamma(R)$ is planar, the (minimum) degree of vertices of $\Gamma(R)$ is at most five. So $\prod_{i=1}^{k}\left(p_{i}^{\alpha_{i}}-2 p_{i}^{\beta_{i}}\right) \leq 5$. Therefore either $\Gamma(R)$ is an empty graph or there exits a non negative integer $r \geq 0$ such that $R$ is isomorphic to one of the following rings:

$\mathbb{Z}_{3}^{r} \times \mathbb{F}_{4}, \mathbb{Z}_{3}^{r}, \mathbb{Z}_{3}^{r} \times \mathbb{F}_{4} \times \mathbb{F}_{4}, \mathbb{Z}_{3}^{r} \times \mathbb{Z}_{5}, \mathbb{Z}_{3}^{r} \times \mathbb{Z}_{9}, \mathbb{Z}_{3}^{r} \times D_{2}\left(\mathbb{Z}_{3}\right)$

On the other hand, it is easy to see that if $R$ is isomorphic to one of the above rings, then $\Gamma(R)$ is planar. This completes the proof. 


\section{ChromatiC NUMBER AND CHROMATIC INDEX}

Let $G$ be a graph. A $k$-colouring of the vertices of $G$ is an assignment of $k$ colours to the vertices of $G$ in such a way that no two adjacent vertices receive the same colour. The chromatic number of $G$, denoted by $\chi(G)$, is the smallest number $k$ such that $G$ admits a $k$-colouring.

Let $G$ and $H$ be two graphs. Since the projections $G \times H \longrightarrow G$ and $G \times H \longrightarrow H$ are homomorphisms, we have $\chi(G \times H) \leq \min \{\chi(G), \chi(H)\}$. One of the most challenging open problems in the context of direct products of graphs is Hedetniemi's Conjecture (see [15, Page 51]). The Hedetniemi's conjecture asserts that for all graphs $G$ and $H$,

$$
\chi(G \times H)=\min \{\chi(G), \chi(H)\} .
$$

This conjecture is also known as Lovász-Hedetniemi conjecture. The following theorem shows that this conjecture is true in the special case:

Theorem 11. Let $R$ and $S$ be two finite rings. Then

$$
\chi(\Gamma(R) \times \Gamma(S))=\min \{\chi(\Gamma(R)), \chi(\Gamma(S))\} .
$$

Proof. Let $R$ be as in Assumption 1. First of all, we show that $\chi(\Gamma(R))=f_{1}-$ 1. We have $\chi(\Gamma(R)) \leq \chi\left(\Gamma\left(R_{1}\right)\right)=f_{1}-1$. Now let $a_{i 1}, \ldots, a_{i\left(f_{1}-1\right)}$ be pairwise adjacent vertices of $\Gamma\left(R_{i}\right)$. Then

$$
\begin{aligned}
& \left(a_{11}, a_{21}, \ldots, a_{n 1}\right), \\
& \left(a_{12}, a_{22}, \ldots, a_{n 2}\right), \\
& \quad \vdots \\
& \left(a_{1\left(f_{1}-1\right)}, a_{2\left(f_{1}-1\right)}, \ldots, a_{n\left(f_{1}-1\right)}\right),
\end{aligned}
$$

are pairwise adjacent vertices of $\Gamma(R)$. Therefore $\chi(\Gamma(R)) \geq f_{1}-1$ and hence $\chi(\Gamma(R))=f_{1}-1$. Now let $S=S_{1} \times \ldots \times S_{m}$, where $S_{i}$ is a local ring with maximal ideal $\mathfrak{n}_{i}, g_{i}=\left|S_{i} / \mathfrak{n}_{i}\right|$ and $g_{1} \leq g_{2} \leq \ldots \leq g_{m}$. Then

$$
\left.\chi(\Gamma(R) \times \Gamma(S))=\min \left\{f_{1}-1, g_{1}-1\right\}\right)=\min \{\chi(\Gamma(R)), \chi(\Gamma(S))\} .
$$

This completes the proof.

An $k$-edge colouring of a graph $G$ is an assignment of $k$ colours to its edges so that no two edges incident to same vertex the same colour. The chromatic index of $G$, denoted by $\chi^{\prime}(G)$, is the least $k$ for which $G$ has an $k$-edge colouring. Vizing's Theorem ([29]) gives us a basic way of classifying graphs into two classes. A graph $G$ is of Class 1 if $\chi^{\prime}(G)=\Delta(G)$ and of Class 2 if $\chi^{\prime}(G)=\Delta(G)+1$. The following theorem determines the class of $\Gamma(R)$.

Theorem 12. Let $R$ be a finite ring. Then

$$
\chi^{\prime}(\Gamma(R))= \begin{cases}\Delta(\Gamma(R))+1 & \text { if }\left|R^{*}\right| \text { is odd, } \\ \Delta(\Gamma(R)) & \text { if }\left|R^{*}\right| \text { is even, }\end{cases}
$$


Proof. If $G=\Gamma(R)$ is a graph of odd order, then $|E(G)|>\Delta(G)\left\lfloor\frac{V(G)}{2}\right\rfloor$, that is $G$ is overfull graph. Therefore [10, Corollary 8.18] implies that $G$ is of Class 2 .

Now let $R$ be as in Assumption 1. If $G$ is a graph of even order, then there exits $1 \leq i \leq n$ such that $\Gamma\left(R_{i}\right)$ is a graph of even order. By [25, Corollary 2.7], $\Gamma\left(R_{i}\right)$ is of Class 1 and [27, Theorem 4.4] implies that $G$ is of Class 1.

\section{ISOMORPHISMS}

The adjacency matrix of a graph is the matrix with rows and columns indexed by its vertices such that the $(i, j)$-entry is equal to 1 if vertices $i$ and $j$ are adjacent and 0 otherwise. The eigenvalues of a graph are eigenvalues of its adjacency matrix, and the spectrum of a graph is the collection of its eigenvalues together with multiplicities. If $\lambda_{1}, \ldots, \lambda_{k}$ are distinct eigenvalues of a graph $G$ and $m_{1}, \ldots, m_{k}$ the corresponding multiplicities, then we denote the spectrum of $G$ by

$$
\operatorname{Spec}(G)=\left(\begin{array}{ccc}
\lambda_{1} & \ldots & \lambda_{k} \\
m_{1} & \ldots & m_{k}
\end{array}\right)
$$

that is, the multiset of eigenvalues of the adjacency matrix of $G$. For example, for $n>1$, we have

$$
\operatorname{Spec}\left(K_{n}\right)=\left(\begin{array}{cc}
n-1 & -1 \\
1 & n-1
\end{array}\right) .
$$

The energy of a graph $G$ with $n$ vertices and with eigenvalues $\lambda_{1}, \ldots, \lambda_{k}$ is defined as

$$
\varepsilon(G)=\sum_{i=1}^{k}\left|\lambda_{i}\right| .
$$

The energy of a graph was introduced in [13] in the context of mathematical chemistry. Since then it has been studied extensively; see for example [14], [16], [19] and [23]. As usual, we use $\mathbb{N}$ for the set of all natural numbers.

Theorem 13. Let $n(i), m(j) \in \mathbb{N} \backslash\{1\}$, where $1 \leq i \leq r$ and $1 \leq j \leq s$. If $\prod_{i=1}^{r} K_{n(i)} \cong \prod_{j=1}^{s} K_{m(j)}$, then $r=s$ and there is a permutation $\sigma$ of $\{1,2, \ldots, r\}$ such that $n(i)=m(\sigma(i))$ for all $1 \leq i \leq r$.

Proof. Let $G=\prod_{i=1}^{r} K_{n(i)}, H=\prod_{j=1}^{s} K_{m(j)}, a=\prod_{i=1}^{r}(n(i)-1)$ and $b=$ $\prod_{j=1}^{s}(m(j)-1)$. Since $G$ is $a$-regular and $H$ is $b$-regular we must have $a=b$. On the other hand, $\varepsilon(G)=2^{r} a$ and $\varepsilon(H)=2^{s} b$. So $r=s$. Without loss of generality, we can assume that

$$
\begin{aligned}
n(1) & \leq \cdots \leq n(r), \\
m(1) & \leq \cdots \leq m(r) .
\end{aligned}
$$


We prove that $n(i)=m(i)$ for all $1 \leq i \leq r$. Since $(-1)^{r-1}(n(1)-1) \in \operatorname{Spec}(G)$ and $(-1)^{r-1}(m(1)-1) \in \operatorname{Spec}(H)$, we must have $n(1)=m(1)$.

On the contrary let $i$ be the smallest number such that $n(i) \neq m(i)$. Without loss of generality, we can assume that $m(i)<n(i)$. Let $C \subseteq\{1,2, \ldots, i-1\}$. Let $X$ and $Y$ be the multisets of $(-1)^{r-|C|} \prod_{i \in C}(n(i)-1)$ and $(-1)^{r-|C|} \prod_{i \in C}(n(i)-1)$, respectively. By the assumption, $|X|=|Y|$ and hence as multisets we have $\operatorname{Spec}(G) \backslash$ $X=\operatorname{Spec}(H) \backslash Y$. Therefore $(-1)^{r-1}(m(i)-1) \in \operatorname{Spec}(H) \backslash Y$. So there exists a subset $C \subseteq\{1,2, \ldots, r-1\}$ such that $C \nsubseteq\{1,2, \ldots, i-1\}$ and $(-1)^{r-1}(m(i)-1)=$ $(-1)^{r-|C|} \prod_{i \in C}(n(i)-1)$, which is a contradiction.

We note that the above theorem is proved in [20], when $n(i), m(j) \in \mathbb{N} \backslash\{1,2\}$.

Lemma 1. Let $R$ be as in Assumption 1. Let $\Gamma(R)$ be a non empty and let $x, y$ be two vertices of $\Gamma(R)$. Then the following are equivalent:

(1) $x-y \in J(R)$,

(2) $N(x)=N(y)$.

Proof. (1) $\Longrightarrow(2)$ Let $u \in N(x)$. Then $u-x \in U(R)$. We claim that $u \in N(y)$. Suppose on the contrary that $u \notin N(y)$. Then there is a maximal ideal $\mathfrak{m}$ of $R$ such that $u-y \in \mathfrak{m}$. Therefore $u-x=(u-y)+(y-x) \in \mathfrak{m}$, which is a contradiction. It follows that $N(x) \subseteq N(y)$. A similar argument shows that $N(y) \subseteq N(x)$.

(2) $\Longrightarrow$ (1) Suppose that $x-y \notin J(R)$. Let $x=\left(x_{1}, \ldots, x_{n}\right)$ and $y=\left(y_{1}, \ldots, y_{n}\right)$. So there exists $1 \leq i \leq n$ such that $x_{i}-y_{i} \notin \mathfrak{m}_{i}$. Since $f_{1} \neq 2$, there exists $z_{j} \in R_{j}$ such that $y_{j}-z_{j} \notin \mathfrak{m}_{i}$ for all $1 \leq j \neq i \leq n$. Let

$$
z=\left(z_{1}, \ldots, z_{i-1}, x_{i}, z_{i+1}, \ldots, z_{n}\right)
$$

Then $z \in N(y) \backslash N(x)$, which is a contradiction.

We are now in a position to prove the main result of this section.

Theorem 14. Let $R$ and $S$ be two finite rings such that $\Gamma(R)$ is non empty. If $\Gamma(R) \cong \Gamma(S)$, then the following hold:

(1) $R / J(R) \cong S / J(S)$,

(2) $|J(R)|=|J(S)|$,

(3) $|R|=|S|$.

Proof. (1) Let $f: \Gamma(R) \longrightarrow \Gamma(S)$ be a graph isomorphism. Define

$$
\begin{aligned}
\bar{f}: \Gamma(R / J(R)) & \longrightarrow \Gamma(S / J(S)) \\
x+J(R) & \longmapsto f(x)+J(S)
\end{aligned}
$$

The above lemma implies that $\bar{f}$ is an isomorphism. By [7, Proposition 1.10] or [21, Theorem 3.5], the rings $R / J(R)$ and $S / J(S)$ are direct product of finite fields. Since two finite fields are isomorphic if and only if they have the same number of elements, Theorem 13 implies that $R / J(R) \cong S / J(S)$. 
(2) Let $R$ be as in Assumption 1 and let $S=S_{1} \times \cdots \times S_{m}$, where $S_{i}$ is a local ring with maximal ideal $\mathfrak{n}_{i}$ such that $\left|S_{1} / \mathfrak{n}_{1}\right| \leq \ldots \leq\left|S_{m} / \mathfrak{n}_{m}\right|$. By Part (1), we have $R / J(R) \cong S / J(S)$. It follows that

$$
\Gamma\left(R_{1} / \mathfrak{m}_{1}\right) \times \cdots \times \Gamma\left(R_{n} / \mathfrak{m}_{n}\right) \cong \Gamma\left(S_{1} / \mathfrak{n}_{1}\right) \times \cdots \times \Gamma\left(S_{m} / \mathfrak{n}_{m}\right) .
$$

In view of Part (1), we have $n=m$ and $\left|R_{i} / \mathfrak{m}_{i}\right|=\left|S_{i} / \mathfrak{n}_{i}\right|$ for all $1 \leq i \leq n$. Then $\left(\left|R_{i}\right|-\left|\mathfrak{m}_{i}\right|\right) /\left|\mathfrak{m}_{i}\right|=\left(\left|S_{i}\right|-\left|\mathfrak{n}_{i}\right|\right) /\left|\mathfrak{n}_{i}\right|$ for all $1 \leq i \leq n$. It follows that $\left|R^{*}\right| /|J(R)|=$ $\left|S^{*}\right| /|J(S)|$. Since $\left|R^{*}\right|=\left|S^{*}\right|$, we have $|J(R)|=|J(S)|$.

(3) Follows easily from the Parts (1) and (2).

We recall that a ring $R$ is called reduced if $R$ has no non-zero nilpotent elements.

Corollary 1. Let $R$ and $S$ be two finite rings such that $R$ is a reduced ring and $\Gamma(R)$ is non empty. If $\Gamma(R) \cong \Gamma(S)$ as graphs, then $R \cong S$ as rings.

Proof. Since $R$ is reduced, we have $\operatorname{Nil}(R)=0(\operatorname{Nil}(R)$ is the intersection of prime ideals of $R$ ). On the other hand, since $R$ is finite, every prime ideal of $R$ is maximal. Hence $J(R)=\operatorname{Nil}(R)=0$. By Theorem $14(2)$, we have $J(S)=0$. Now the assertion follows from Theorem 14(1).

\section{ACKNOWLEDGEMENT}

I would like to thank the referee for the careful review and the valuable comments. I would also like to thank the Center of Excellence for Mathematics, Shahrekord university for financial support.

\section{REFERENCES}

[1] G. Aalipour and S. Akbari, "Some properties of a Cayley graph of a commutative ring," Comm. Algebra, vol. 42, pp. 1582-1593, 2014, doi: 10.1080/00927872.2012.745866.

[2] G. Aalipour and S. Akbari, "On the Cayley graph of a commutative ring with respect to its zerodivisors," Comm. Algebra, vol. 44, pp. 1443-1459, 2016.

[3] S. Akbari and A. Mohammadian, "On the zero-divisor graph of a commutative ring," J. Algebra, vol. 274, pp. 847-855, 2004, doi: 10.1016/S0021-8693(03)00435-6.

[4] R. Akhtar, M. Boggess, T. Jackson-Henderson, I. Jiménez, R. Karpman, A. Kinzel, and D. Pritikin, "On the unitary Cayley graph of a finite ring." Electron. J. Combin., vol. 16 \#R117, pp. 1-13, 2009.

[5] D. F. Anderson, A. Frazier, A. Lauve, and P. S. Livingston, "The zero-divisor graph of a commutative ring, II," Lecture Notes in Pure and Appl. Math., vol. 220, pp. 61-72, 2001.

[6] D. F. Anderson and P. S. Livingston, "The zero-divisor graph of a commutative ring," J. Algebra, vol. 217, pp. 434-447, 1999, doi: 10.1006/jabr.1998.7840.

[7] M. F. Atiyah and I. G. MacDonald, Introduction to Commutative Algebra. Massachusetts: Addison-Wesley, 1969.

[8] I. Beck, "Coloring of commutative rings," J. Algebra, vol. 116, pp. 208-226, 1988, doi: 10.1016/0021-8693(88)90202-5.

[9] J. A. Bondy and U. S. R. Murty, Graph Theory. New York: Springer-Verlag, 2008.

[10] G. Chartrand and L. Lesniak, Graphs and Digraphs. New York: Chapman and Hall, 2000.

[11] D. Dolžan, "Group of units in a finite ring," J. Pure Appl. Algebra, vol. 170, pp. 175-183, 2002, doi: 10.1016/S0022-4049(01)00080-9. 
[12] R. P. Grimaldi, "Graphs from rings. In: Proceedings of the 20th Southeastern Conference on Combinatorics, Graph Theory, and Computing (Boca Raton, FL, 1989)." Congr. Numer., vol. 71, pp. 95-103, 1990.

[13] I. Gutman, "The energy of a graph," Ber. Math. Stat. Sekt. Forschungszent. Graz, vol. 103, pp. $1-22,1978$.

[14] I. Gutman, The energy of a graph: old and new results. In: A. Betten, A. Kohnert, R. Laue and A. Wassermann, Editors, Algebraic Combinatorics and Applications. Berlin: Springer-Verlag, 2001.

[15] P. Hell and J. Nešetřil, Graphs and Homomorphisms. New York: Oxford University Press, 2004.

[16] A. Ilić, "The energy of unitary Cayley graphs," Linear Algebra Appl., vol. 431, pp. 1881-1889, 2009, doi: 10.1016/j.laa.2009.06.025.

[17] W. Imrich and S. Klavžar, Product Graphs. New York: John Wiley and Sons, 2000.

[18] D. Kiani and M. Molla Haji Aghaei, "On the unitary Cayley graph of a ring," Electron. J. Combin., vol. 19, no. 2 \#P10, pp. 1-10, 2012.

[19] D. Kiani, M. Molla Haji Aghaei, Y. Meemark, and B. Suntornpoch, "Energy of unitary Cayley graphs and gcd-graphs," Linear Algebra Appl., vol. 435, pp. 1336-1343, 2011, doi: 10.1016/j.laa.2011.03.015.

[20] V. Koubek, J. Nešetřil, and V. Rödl, "Representing groups and semigroups by products in categories of relations," Algebra Universalis, vol. 4, pp. 336-341, 1974, doi: 10.1007/BF02485746.

[21] T. Y. Lam, A First Course in Noncommutative Rings. New York: Springer-Verlag, 2000.

[22] C. Lanski and A. Maróti, "Ring elements as sums of units," Cent. Eur. J. Math., vol. 7, no. 3, pp. 395-399, 2009, doi: 10.2478/s11533-009-0024-5.

[23] X. Liu and S. Zhou, "Spectral properties of unitary Cayley graphs of finite commutative rings," Electron. J. Combin., vol. 19, no. 4 \#P13, pp. 1-19, 2012.

[24] H. R. Maimani, M. R. Pournaki, A. Tehranian, and S. Yassemi, "Graphs attached to rings revisited," Arab. J. Sci. Eng., vol. 36, pp. 997-1011, 2011, doi: 10.1007/s13369-011-0096-y.

[25] B. Mohar and T. Pisanski, "Edge-coloring of a family of regular graphs," Publ. Inst. Math., vol. 33, pp. 157-162, 1983.

[26] S. N. Nam, "Finite local rings of order $\leq 16$ with nonzero Jacobson radical," Korean J. Math., vol. 21, pp. 23-28, 2013, doi: 10.11568/kjm.2013.21.1.23.

[27] T. Pisanski, J. Shawe-Taylor, and B. Mohar, "1-factorization of the composition of regular graphs," Publ. Inst. Math., vol. 33, pp. 193-196, 1983.

[28] P. K. Sharma and S. M. Bhatwadekar, "A note on graphical representation of rings," J. Algebra, vol. 176, pp. 124-127, 1995, doi: 10.1006/jabr.1995.1236.

[29] V. G. Vizing, "On an estimate of the chromatic class of a p-graph," Diskret. Analiz, vol. 3, pp. 25-30, 1964.

[30] D. B. West, Introduction to Graph Theory (Second Edition). Englewood Cliffs, NJ: Prentice-Hall, 2000.

Author's address

Ali Reza Naghipour

Sharekord University, Department of Mathematics, P.O. Box 115, Shahrekord, Iran

E-mail address: naghipouresci.sku.ac.ir 